\title{
La conception du développement de la France coloniale en Afrique Noire : Quelques réflexions à propos de l'APAD
}

\section{Ulrike Schuerkens}

\author{
(2) OpenEdition \\ Journals \\ Édition électronique \\ URL : http://journals.openedition.org/apad/3193 \\ DOI : 10.4000/apad.3193 \\ ISSN : 1950-6929 \\ Éditeur \\ LIT Verlag \\ Édition imprimée \\ Date de publication : 1 juin 1993 \\ Référence électronique \\ Ulrike Schuerkens, « La conception du développement de la France coloniale en Afrique Noire: \\ Quelques réflexions à propos de l'APAD », Bulletin de l'APAD [En ligne], 5 | 1993, mis en ligne le 04 juin \\ 2008, consulté le 07 septembre 2020. URL : http://journals.openedition.org/apad/3193 ; DOI : https:// \\ doi.org/10.4000/apad.3193
}

Ce document a été généré automatiquement le 7 septembre 2020

Bulletin de I'APAD 


\title{
La conception du développement de la France coloniale en Afrique Noire : Quelques réflexions à propos de l'APAD
}

\author{
Ulrike Schuerkens
}

1 L'intitulé de notre association, et ainsi, les termes "Association Euro-Africaine du Changement Social et du Développement" méritent quelques réflexions. Cette terminologie serait pauvre si elle s'appliquait seule à l'espace culturel de l'Europe et de l'Afrique. En effet, il s'agit de beaucoup plus. L'Afrique, et, notamment, l'Afrique Noire avait des relations privilégiées avec l'Europe depuis le début de ce siècle, et en ce qui nous concerne, avec la France. Ces rapports intenses reposaient sur un ensemble théorique et idéologique qui fut appliqué dans cette politique coloniale et qui définissait le caractère spécifique de ces liens. Nous développerons ainsi dans les pages qui suivront les aspects conceptuels et idéologiques ${ }^{1}$ de cette approche liant l'Afrique et la France, et, étant à la base de l'ensemble de la politique du développement colonial, et, en partie, au moins, des efforts actuels. Ceci nous amènera ensuite à insister sur la particularité du changement social en Afrique et à redéfinir le genre d'approche qui nous permettra d'analyser ces processus de transformation ${ }^{2}$.

Les différences de l'humanité selon la pensée coloniale

2 Les idées évolutionnistes de la fin du siècle dernier et du début de ce siècle, et surtout celles de Le Bon, Spencer, et Darwin trouvaient un écho favorable dans le domaine de la politique coloniale. A la fin du XIXème siècle et au début du XXème siècle, un nombre considérable d'ouvrages reprenant l'un ou l'autre de ces arguments fut publié. En France, la supériorité de la civilisation française était incontestée. L'argument de la supériorité intellectuelle de la culture française reposait sur la conviction qu'il existait, d'une part, des peuples civilisés et, d'autre part, des peuples non-civilisés. En ce qui concerne la première catégorie, leur progrès ne semblait pas être mis en doute. Quant à 
la deuxième catégorie, elle n'arriverait pas à sortir de son système culturel et social, sans une intervention extérieure :

"(...) laissées à elles-mêmes, elles en seraient restées toujours et immuablement au même point et (...) pour parvenir plus haut (...) il faut (...) qu'une main leur soit tendue et les tire sur le chemin qui monte". (cf. ALLIER -1927-225).

Selon l'auteur, l'ensemble de cette population avait besoin d'atteindre un autre niveau. Une élite devait être formée en même temps. Les étapes que les races supérieures auraient franchies pendant plusieurs siècles ne pourraient l'être par ces populations en quelques décades. L'identité foncière de tous les hommes, que certains philosophes soutenaient à l'époque, n'était pas niée par Allier. Néanmoins, selon lui, ces identités s'opposaient. Il serait donc vain de croire à la transformation de la première à l'image de la seconde.

Cependant, les missionnaires travaillant parmi ces populations insistaient sur le fait que cette humanité n'est qu'une

"(...) dans son essence et une dans sa destinée. Son œuvre (du missionnaire, US) est bien réellement de faire rentrer dans la famille humaine les enfants arriérés qui, à force d'être arriérés, avaient l'air de ne plus être de la famille" (cf ALLIER -1927-289).

5 Les remarques de Doucet (1926) vont dans le même sens. Selon lui, l'acquisition des colonies obligeait la France à multiplier ses efforts pour que les populations de ces territoires ressemblent le plus possible à celles de la métropole. Ces populations autochtones devaient acquérir la culture française, l'âme française afin de

"(...) poursuivre activement la formation et la croissance, dans toutes celles de nos possessions où la nature n'y met pas d'obstacle, de nouvelles France calquées sur l'image de la métropole". (cf. DOUCET, 1926-11).

Or, il existait à l'époque des colonies

"(...) à population très voisine de l'état de nature et des colonies à population plus ou moins civilisée" (cf. 13).

Donc, selon Doucet, les premières resteraient pendant longtemps dans le sillage des civilisations européennes, qui, tout en possédant une civilisation supérieure, disposaient, de ce fait, du droit d'en faire bénéficier les autres (cf. 42). Harmand, le père fondateur de la politique d'association formulait ce droit en ces termes :

"Le droit que s'arroge le conquérant repose, nous l'avons dit et il faut y insister, sur la conscience qu'il a des supériorités, sur la conviction que sa domination est utile et bonne, sur sa volonté d'en fournir la preuve" (cf. HARMAND 1910-170).

La politique d'association, succédant à la politique de domination pratiquée par la France au début de l'ère coloniale, se caractérisait, selon Harmand, par une utilité réciproque, et non plus par la force. Dans sa forme idéale, elle ressembla à la politique coloniale de l'Angleterre. L'amélioration de la situation matérielle et sociale des indigènes devenait le but principal. Néanmoins, les Français devaient les faire évoluer de telle sorte qu'ils maintiendraient leurs places, leurs fonctions et leurs rôles dans leurs systèmes culturels. Le respect de leurs traditions et de leurs habitudes servirait à atteindre ces objectifs. La réalisation de cette politique d'association demandait donc une connaissance approfondie des populations autochtones qui ne pouvait être acquise que par un contact prolongé. C'est ainsi qu'elle assurerait la conciliation des droits des vainqueurs et les intérêts des populations, des vaincus, et ainsi des 
"(...) plus favorisées avec leurs besoins les plus nécessaires et les devoirs que les vainqueurs ont assumés en imposant aux plus faibles le poids de leur supériorité". (cf. HARMAND -1910-169).

\section{française qui semblait ainsi être le champ privilégié}

"(...) d'accélérer la marche vers le progrès des masses humaines encore enlisées dans le passé, de faire entrer en d'autres termes les sociétés primitives ou attardées dans l'orbite de la Civilisation". (cf. GlRARDET-1972-91).

Pourtant, pendant l'entre-deux-guerres, ces conceptions commençaient à être remplacées par celles de chocs culturels. Peu à peu, la distinction fondamentale entre civilisation supérieure et civilisation inférieure disparaissait du vocabulaire politique. C'est ainsi que la subordination du colonisé au colonisateur faisait place à une conception visant des rapports différents fondés selon le discours sur une égalité des droits et des devoirs.

La conception du développement et la politique coloniale française

\section{l'Angleterre, l'idée de la colonisation ne reposait pas sur un concept unique, mais} comme le constatait Girardet (1972) :

"(...) sur un système (...) plus large de préjugés, de croyances et d'habitudes mentales, liées à la conviction très profonde et à peu près unanimement partagée de l'évidente primauté de la civilisation européenne, - en vérité la seule Civilisation, - par rapport à toutes les autres sociétés humaines. (...) il faut insister sur le fait que cette conviction dépasse très largement les oppositions politiques et les débats idéologiques ou confessionnels" (cf. 89).

Et l'auteur ajoutait :

"L'idée d'une hiérarchie, d'un ordre de valeurs dans l'échelle des communautés humaines constitue un postulat de base que bien peu, apparemment, songent à mettre en cause (...)" (cf. 90).

13 La France n'a donc jamais connu une élaboration théorique et méthodique semblable à celle de Lord Lugard en Angleterre dont le cadre théorique prétendait être applicable à toutes les colonies anglaises ${ }^{3}$. La politique coloniale de la France était au contraire imprégnée des idées provenant d'auteurs d'horizons différents. Parmi eux, sans doute, Jules Harmand était-il le plus important. Ses conceptions influencèrent largement la politique coloniale de la France jusqu'aux années 1940. Harmand était le père fondateur de la politique d'associations de la France. Il la définissait de la manière suivante :

"Nous avons le devoir de maintenir notre domination sur nos sujets. Seule elle peut assurer l'ordre, la prévoyance, la puissance économique. Mais nous devons pratiquer avec eux une Politique d'Association impliquant le respect scrupuleux de leurs coutumes, l'administration indirecte, l'entraide économique, le développement intellectuel et technique. Il y a là une sorte de contrat à l'avantage des deux parties". (cité selon DESCHAMPS-1953-149).

14 Delafosse, pendant longtemps administrateur colonial, conçut la mission de la France et le genre d'administration directe pratiqué par elle en ces termes :

"L'évolution devra être progressive et les institutions conservées tant qu'elles seront_vivantes; nous ne devrons intervenir que discrètement, sauf en matière économique où nous dirigerons les chefs, mal préparés à ce rôle". (cité selon DESCHAMPS -1953-166).

15 Cependant, l'administration directe que la France pratiquait ne se restreignait pas aux seules fonctions des chefferies. Elle concernait, en principe, tous les domaines de la vie 
sociale : les coutumes, l'organisation politique, les règles juridiques, l'enseignement, les activités professionnelles etc... Néanmoins, l'idée de Delafosse persistait que l'instruction et l'éducation des chefs aideraient à former une élite qui" (...) tout en venant à nous, ne quittera pas son peuple". (cité selon DESCHAMPS - 1953-222).

La politique coloniale et même celle plus récente envers les pays de l'Afrique francophone, étant donné qu'une seule conception théorique et pratique n'existait pas, se caractérisaient par un mouvement au jour le jour, guidé par des nécessités du moment ou par les tendances théoriques et politiques à la mode ${ }^{4}$. Doucet caractérisa dans ses "Commentaires sur la Colonisation" (1926) cette politique coloniale en ces mots :

"La politique coloniale est l'art d'organiser et d'outiller, suivant les méthodes modernes, un pays non civilisé, peu civilisé, ou possédant une civilisation très différente de la nôtre, dans le but d'accroître sa richesse et de servir, par contre-coup, les intérêts du peuple colonisateur". (cf. 42).

Harmand définissait la politique de développement liée à sa théorie d'association de cette manière :

"Utilitaire encore dans ses résultats, elle ne produit tous ses effets et ne devient le meilleur de tous les calculs que si elle est vivifiée par des inspirations d'ordre moral et par la conviction que le conquérant en assumant la tutelle des populations, prend aussi charge de leurs âmes, de leurs besoins intellectuels et de leurs sentiments ataviques, et l'engagement de les satisfaire (...)". (cf. HARMAND -1910-158).

La difficulté de cette politique consista selon l'auteur dans l'interprétation réelle sur le terrain:

"Mal comprise, mal pratiquée, elle a pu être interprétée par chacun dans le sens qui convenait à ses tendances ou à ses opinions et d'une manière parfois opposée aux observations et aux réflexions qui l'avaient dictée" (cf. 159).

Les réflexions de Doucet vont dans le même sens :

"La politique d'association est vague et réaliste. Elle consiste à concilier et à harmoniser les intérêts des deux types humains en présence, le colonisateur et le colonisé ; elle consiste au fond, à n'avoir pas de principes, à ne considérer en tout que des cas particuliers, bref à faire de l'opportunisme. Et cela n'est peut-être pas si ridicule. Cette politique du juste milieu qu'est l'opportunisme a été pratiquée de tout temps, notamment par la France qui a su, dans la majeure partie de ses entreprises extérieures, éviter les excès d'autoritarisme comme les excès de sentimentalisme" (cf. DOUCET - 1926-48).

20 La généralité de certaines de ces constatations, poussaient les administrateurs sur place à interpréter eux-mêmes la politique à suivre. Malgré la dépendance de la France des gouverneurs ou représentants dans les différents territoires africains, due à la centralisation caractéristique de la politique administrative française, celle-ci leur permettrait de disposer d'une marge de manœuvre assez vaste. Néanmoins, leurs pouvoirs étaient limités dans la mesure où certaines activités exigeaient le consentement $\mathrm{du}$ ministère des colonies ou du gouvernement français. Les administrateurs sur place avaient, pourtant, la possibilité de définir une politique concrète. Ces initiatives n'ont pas toujours trouvé l'approbation de Paris. C'est ainsi que des décrets ou des propositions de décrets pouvaient orienter l'administration coloniale. Dans la mesure où ces propositions n'avaient pas le caractère d'une loi, ils pouvaient servir en tant que guide dans la politique courante de ces pays.

21 La transposition de ces idées dans une politique concrète reposait sur différentes conceptions. La société, et dans ce sens les idées de H. Spencer ${ }^{5}$ ont été largement 
acceptées, possédait selon les auteurs une structure organique, composée d'individus disposant des caractéristiques nationales héréditaires. On en déduisait que le présent ne pouvait qu'être compris en tenant compte du passé. Une séparation totale, même heuristique, n'était pas concevable. La conclusion était, ainsi, que les nations comme les individus possédaient des traits typiques uniquement compréhensibles à partir de l'analyse du changement de ceux-ci. Ces traits étaient caractérisés, de plus, comme non-modifiables brusquement, mais lentement et graduellement (cf. BETTS - 1961-24).

À cette idée était liée la pensée de varier la pratique coloniale selon les caractéristiques géographiques, ethniques et l'état du développement social du groupe en question. La conception de base était qu'une coopération étroite entre le pouvoir colonial et la population autochtone était nécessaire et même obligatoire. Dans le meilleur des cas, cette coopération était atteinte en acceptant les institutions locales. A l'Européen incombait la responsabilité de garantir les bénéfices matériels représentant certaines valeurs pour la culture autochtone. Selon cette idée, la population autochtone, de son côté, en réfléchissant sur les valeurs qui lui étaient imposées, commencerait, ensuite, à coopérer plus étroitement avec les Européens.

Améliorer le statut matériel et culturel des populations autochtones exigeait, par conséquent, l'instauration des institutions adaptées à leur cadre social. En plus, afin de réaliser plutôt les buts économiques de la France, certaines institutions françaises ou largement inspirées des modèles français devaient être imposées à côté ou à la place des institutions des populations autochtones. Si l'on voulait que ces institutions puissent s'adapter au cadre offert par la culture autochtone, on oubliait souvent que les règles régissant la vie de ses populations étaient peu connues. Il s'agissait, ainsi, dans l'œuvre coloniale de la France, d'une restructuration des sociétés autochtones selon un modèle plus ou moins réussi, et offert par l'héritage culturel français ${ }^{6}$.

L'application concrète paraissait facile, à condition que certaines idées fussent retenues : d'abord, le respect mutuel entre la population autochtone et la population française devait être assuré afin d'atteindre le plus vite possible le but visé, et notamment une étroite coopération entre les deux sociétés. Ensuite, les deux groupes devaient accepter des responsabilités communes tout en améliorant la situation du territoire. Enfin, on exigeait une administration locale suffisamment développée pour que le développement économique et social puisse se dérouler sans heurts majeurs.

Or, l'administration française considérait que le contact entre les deux groupes en question avait une influence importante sur le changement des sociétés autochtones. Ainsi Deschamps constata en 1953 :

"Les chefferies, les hiérarchies traditionnelles, l'autorité des anciens, sur lesquelles repose notre administration, voient peu à peu leur influence diminuer au profit des enrichis, des travailleurs, des évolués formés dans nos écoles". (cf. DESCHAMPS -1953-173).

On peut dire, à ce point de l'analyse, que le cadre théorique et méthodique de la politique coloniale française reposait sur la conviction qu'il fallait développer les populations des colonies en posant comme axiome la supériorité de la culture française. Les différents auteurs que nous avons cités démontrent clairement cette tendance de la France à utiliser sa culture en tant que moyen civilisateur. Cette approche était basée sur la conviction que la culture française était supérieure aux cultures autochtones. Le contact entre les deux cultures, étant donné que la culture française, au moins dans les pays francophones de l'Afrique Noire, était beaucoup plus 
complexe que celle des populations autochtones, ne pouvait que favoriser l'évolution de la société autochtone vers une proximité de plus en plus étroite avec certains éléments du modèle français. Le pouvoir inégal et les connaissances et buts de l'œuvre civilisatrice française influençaient ce développement à un tel point que certains auteurs ont pu parler d'une occidentalisation des mœurs au moins parmi les groupes dirigeants de ces pays ${ }^{7}$.

Le développement de l'Afrique Noire et la France

En guise de conclusion, nous insisterons sur le fait que le but de cette politique coloniale était de créer des habitudes, des notions et des institutions acceptables par les systèmes sociaux, culturels et économiques des populations autochtones, tout en considérant les relations de force existantes qui privilégiaient la France dans $l^{\prime}$ introduction de ses règlements ${ }^{8}$. Le processus de changement continuel créa donc des institutions semblables à celles de la Métropole; des faits accomplis qui, la plupart du temps, ne pouvaient être gérés qu'avec le savoir-faire et les connaissances de la France. L'indépendance de ces pays et la continuité des relations avec la France s'intégraient parfaitement à cette conception du développement. Les frictions et ruptures apparaissant pendant toute la période d'administration coloniale de la France ne pouvaient à la plupart du temps être assumées que par le modèle social, culturel, politique et économique proposé. L'indépendance voyait les pays de l'Afrique francophone pourvus de tout un héritage culturel et social caractéristique pour la France.

Cependant, le mélange actuel entre les deux cultures se révèle être plein d'ambiguïté, et ceci, dû aux faits hérités de la colonisation. Il n'est pas de notre propos de révéler l'éventail des dysharmonies 9. La colonisation considérée comme la transmission d'un héritage culturel appartenant à un autre groupe social ne pouvait se fonder, et ceci, à cause de ses fortes tendances civilisatrices, sur la reconnaissance sans a priori des pratiques africaines. Les pays occidentaux persuadés de leur mission civilisatrice avaient des buts différents. Il ne s'agissait plus seulement d'explorer ou d'étudier des cultures étrangères, mais d'essayer de transmettre des connaissances et un savoir-vivre que l'Africain ne possédait pas. Dans ce sens, la colonisation n'était ni une rupture, ni une parenthèse - pour reprendre le titre du livre édité par Piault (1987) -, car ces pratiques coloniales et leurs effets avaient des influences sur les structures sociales de ces sociétés.

La conception du développement de la France aboutissait ainsi dans les quelques décades de la colonisation française à de multiples situations caractérisées par cette interaction entre deux conceptions de sociétés différentes et reflétant l'incompréhension mutuelle. Pourtant, on commence à admettre que ce développement poursuit un genre de syncrétisme, entre, d'une part, des éléments venant des sociétés autochtones, et, d'autre part, ceux du modèle occidental ${ }^{10}$. La problématique qui se posera, par conséquent, - et avec ceci nos propos rejoindront les objectifs de l'APAD -, résidera dans la nécessité absolue d'une description détaillée de ce genre de développement, élaboré et pratiqué par la France, et de ses effets inattendus, imprévisibles et retrouvant souvent ses origines dans les cultures des populations autochtones.

Il ne devrait donc plus être question de chercher des voies de développement possibles, mais de mettre l'accent sur l'analyse de certains faits accomplis et d'étudier en profondeur leurs effets secondaires ${ }^{11}$. La discipline et, en particulier l'africanisme, 
n'avanceront que s'ils se rendent compte des a priori créés par la colonisation qui déterminent le présent et également l'avenir de ces sociétés. L'analyse des problématiques actuelles doit, par conséquent, passer par cette compréhension d'une logique coloniale, sans pour autant adhérer à l'ensemble idéologique et théorique qu'impliquait cette œuvre civilisatrice. L'anthropologie sera bien conseillée, si elle veut progresser dans son domaine, de se baser sur l'ensemble des recherches effectuées pendant les dernières décades, tout en tenant compte d'une colonisation qui mettait le fondement à une restructuration profonde de ces sociétés, à laquelle nous sommes confrontés aujourd'hui, et que nous avons des difficultés à saisir avec les approches traditionnelles de la discipline. Notre but est atteint dans la mesure où il permet de révéler la genèse historique et idéologique d'un processus encore difficile à comprendre.

\section{BIBLIOGRAPHIE}

Allier Raoul. 1927. Le non-civilisé et nous. Différence irréductible ou identité foncière ? Paris, Payot, 317 p.

Balandier Georges. 1951. "La situation coloniale : Approche théorique". Cahiers Internationaux de Sociologie, XI, 44-79.

Balandier Georges. 1985. Le détour. Pouvoir et modernité. Paris, Fayard, 268 p.

Bayart Jean-François. 1984. La politique africaine de François Mitterand. Paris, Khartala, 155 p.

Bayart Jean-François. 1989. L'État en Afrique. La politique du ventre. Paris, Fayard, 439p.

Bessis Sophie. 1983. La dernière frontière : les tiers-mondes et la tentation de l'Occident. Paris, J.-C. Lattes, 299 p.

Betts F. Raymond. 1961. Assimilation and Association in French colonial Theory, 1890-1919. New-York, London, Columbian University Press, ix-244 p.

Betts F. Raymond. 1985. Uncertain Dimensions: Western Overseas Empires in the Twentieth Century. Oxford, Oxford University Press, $284 \mathrm{p}$.

Boudon Raymond. 1986. L'idéologie ou l'origine des idées reçues. Paris, Fayard, 330 p.

Dahmani Roland. 1983. L'occidentalisation des pays du Tiers-Monde. Mythes et réalités. Alger, Economia, $217 \mathrm{p}$.

Deschamps Hubert. 1953. Les méthodes et doctrines coloniales de la France (du XVI siècle à nos jours). Paris, A. Colin, 222 p.

Doucet Robert. 1926. Commentaires sur la colonisation. Paris, Larose, 141 p.

Duby Georges. . "Histoire sociale et idéologies des sociétés". In : Faire de l'histoire. Nouveaux problèmes. Sous la direction de Jacques Le GOFF et Pierre NORA. Paris, Gallimard, vol. 1, 147-168.

Girardet Raoul. 1972. L'idée coloniale en France de 1871 à 1961. Paris, Brodard et Taupin, xii-267 p.

Harmand Jules. 1910. Domination et colonisation. Paris, Flammarion, 370 p. 
Ingold Tim. 1986. Evolution and Social Life. Cambridge, Cambridge University Press, xv-431 p.

Laszlo Ervin. 1989. La cohérence du réel. Évolution, cœur du savoir. Paris, Gauthiers-Villars, 230 p.

Malinowski Bronislaw. 1970. Les dynamiques de l'évolution culturelle. Paris, Payot, 237 p.

Peel John David Yeadon (ed.). 1972. Spencer, Herbert. On social Evolution. Selected Writings. Chicago and London, The University of Chicago Press, li-270 p.

Piault Marc (ed.). 1987. La Colonisation, rupture ou parenthèse. Paris, L'Harmattan, 326p.

Pons Philippe. 1988. D'Edo à Tokyo : mémoires et modernités. Paris, Gallimard, $458 \mathrm{p}$.

Sahlins Marshall. 1985. Islands of History. Chicago, University of Chicago Press, $191 \mathrm{p}$.

Schuerkens Ulrike. 1990. "Débat théorique et travaux empiriques : Le développement du Tiers-Monde". Communication présentée lors de la Sixième Conférence Générale de l'Association des Instituts de recherche et de formation en matière de développement. Oslo, 27-30 Juin 1990,23 p., à paraître.

Schuerkens Ulrike. 1991a. "L'évolution des sociétés occidentales et le système-monde, XIXe siècle - XXIe siècle : Le cas de la France". Communication présentée lors du Colloque "L'évolution des sociétés occidentales et le système-monde, XIXe siècle -XXIe siècle", Maison des Sciences de l'Homme, Paris, 10-12 Janvier 1991,23 p.

Schuerkens Ulrike. 1992a. "L'administration française au Togo (1919-1940) et l'utilisation de la notion de coutume". Droit et cultures, $\mathrm{N}^{\circ} 23$, sous presse.

Schuerkens Ulrike. 1992b. "The notion of Development in Great-Britain in the XXth Century and some Aspects of its Application in Togoland under British Mandate and Trusteeship".p., (manuscrit non-publié).

Spencer Herbert. 1878-1887. Principes de sociologie. Traduit par M.E. Cazelles, Paris, G. Baillère, 4 vol.

Todorov Tzvetan. 1989. Nous et les autres. La réflexion française sur la diversité humaine. Paris, Seuil, $450 \mathrm{p}$.

Touré Abdou. 1981. La civilisation quotidienne en Côte-d'Ivoire. Procès d'occidentalisation. Paris, Khartala, $279 \mathrm{p}$.

Turner Jonathan H. 1985. Herbert Spencer. A Renewed Appreciation. Beverly Hills, London, New Delhi, Sage Publications, 160 p.

Ziegler Jean. 1988. La victoire des vaincus. Oppression et résistance culturelle. Paris, Seuil, 250 p.

\section{NOTES}

1.Cf. en ce qui concerne la notion d"'idéologie" : Duby - 1974 et Boudon - 1986.

2.Ce texte est issu d'une réflexion provoquée par l'article de J. Copans au Journal des Anthropologues $(46,1992-23 / 32)$ : "Notre anthropologie est-elle bien celle des autres ?... et réciproquement ! ?".

3.Cf. en ce qui concerne la conception du développement de l'Angleterre notre manuscrit (1992-26) : "The notion of Development in Great-Britain in the XX ${ }^{\text {th }}$ Century and some Aspects of its Application in Togoland under British Mandate and Trusteeship" . 
4.Cf. à ce propos Bayart - 1984-100 et suite, et notre contribution au Colloque "L'évolution des société occidentales et le système-monde, XIXe siècle - XXIe siècle". Maison des Sciences de l'Homme, Paris, 10-12 Janvier 1991 : "L'évolution des sociétés occidentales et le système-monde, XIXe siècle - XXIe siècle : Le cas de la France". (23p.). 5.Cf. entre autres Spencer-187811887 ou Peel-1972 qui présente les textes de Spencer concernant la problématique de l'évolution. En ce qui concerne la théorie de l'évolution dans sa forme actuelle, on consultera par exemple Sahlins-1985, Ingold-1986 ou Laszlo-1989.

6.Cf. dans ce sens notre article "L'administration française au Togo (1919-1940) et l'utilisation de la coutume". Droit et culture, $n^{\circ} 23$, sous presse.

7.Cf. à ce propos Touré-1981, Bessis-1983, ou bien Dahmani-1983.

8.Une démonstration de ces différents aspects de la politique coloniale de la France nous amènerait dans un domaine trop vaste pour être traité dans le cadre de cet article. 9.Le lecteur intéressé peut trouver une large démonstration de ces principes dans l'ouvrage de Ziegler-1988.

10.Cf. dans ce sens notre communication lors de la VIème Conférence Générale de l'Association Européenne des Instituts de Recherche en matière de Développement : "Débat théorique et travaux empiriques : le développement du Tiers-Monde." Oslo, 27 au 30 Juin 1990, 23 p., à paraître. Je discute dans cette analyse la problématique des théories du changement social des années 1960 jusqu'aux années 1990 et leur contribution à l'explication actuelle du changement social. Dans une deuxième partie, je propose un cadre théorique qui englobe des facteurs endogènes et exogènes du développement social ainsi que l'aspect diachronique. Pons-1988 utilise une approche semblable en analysant la transformation profonde qu'a connue la société japonaise avec l'introduction d'une économie capitaliste après la première guerre mondiale. L'auteur démontre comment la société japonaise a réagi à cette confrontation à un autre type de société et a réussi finalement à intégrer dans un nouveau code symbolique des éléments venant des deux systèmes sociaux en interaction. 11.Pour donner un exemple de ce genre d'analyse, nous renvoyons le lecteur à l'ouvrage de Bayart-1989.

\section{AUTEUR}

\section{ULRIKE SCHUERKENS}

Paris 